\title{
Research on Rainfall Erosion Model Tests for Loess Slope
}

\author{
Wei Wang ${ }^{1, ~ a}$ and Zhiqing $\mathrm{Li}^{2} \mathrm{~b}^{*}$ \\ ${ }^{1}$ Henan Province Y.L.X Expressway Construction Co. Ltd., Luoyang 471521, China \\ ${ }^{2}$ Key Laboratory of Shale Gas and Geoengineering, Institute of Geology and Geophysics, Chinese \\ Academy of Sciences, Beijing 100029, China \\ a84575366@qq.com, ${ }^{\mathrm{b}}$ lizhiq-2002@163.com \\ * The corresponding author
}

Keywords: Loess landslide; Modifying agent; Ecological slope fixation; Lattice beam

\begin{abstract}
It has been a difficult issue that the surface of side slope can be easily washed out by rainfall, causing water and soil loss and thus landslide. In this paper, taking loess slope as an example, a comprehensive prevention and protection method by ecological slope fixation. The impact on protection effect by the following factors are taken into consideration: mixing amount of different modifying agent, lattice protection methods, rainfall intensity, duration, slope ratio, etc. The rules and evolutionary mechanism of soil mass erosion under these factors and their different combinations are studied. The experiment results indicate that as the mixing amount of modifying agent increases, the anti-erosion ability of ameliorated side slope will be enhanced, and the optimal mixing ratio of modifying agent turns out to be 3\%. Compared with plain soil side slope, the side slope ameliorated by $3 \%$ of modifying agent is less affected by rainfall intensity. As the duration increases, the effect of modifying agent becomes more distinct. By applying the modifying agent and lattice beam independently and jointly, the rain wash resistance and anti-erosion ability of the side slope will both be improved. The modifying agent connects and bonds the soil particles tighter mainly through flocculation, as a result, the water binding and anti-erosion capacity of loess are improved.
\end{abstract}

\section{Introduction}

With the development of economy and society, the scale of highway construction has been expanding, and the ensuing landslide, collapse and debris flow are increasing as well, especially the highway slope's water and soil loss and superficial layer landslide caused by rainfall. These disasters present threats to people and vehicles passing by.

Currently, it is using both engineering protection, such as rockbolt lattice beam and arch protection slope, and ecological protection, for example, the seed-nutriment-soil sacks and alien earth spraying, to protect the highway slopes [1,2]. These combined approaches have achieved some certain protective results but geologic hazads like slope debris flow, landslide and deterioration of the slope's anti-erosion capacity still exist in the long term. The jointly use of earth solidification and vegetation reinforcement technology to protect the shallow slope attracts much attention from scholars at home and abroad. The effects of EN-1 solidification agent on earth structure, soil moisture characteristics such as water holding and water supplying, and stimulated anti-erosion capacity were studies and the results suggested that EN-1 solidification agent can improve the soil stability of loess slope and realize the ecological protection of slope vegetation [3]. The indoor test has been conducted over a new polymer soil stabilizer, showing that the soil stabilizer can significantly improve the anti-erosion capacity and water stability of the modified soil, which means it is better to applied to fields such as soil and water conservation, and ecological protection of clay soil slope [4]. A research made showed that the ability of polyacrylamide to improve the earth's water resistance capacity and anti-erosion capacity lies in the promotion of soil sedimentation and the enhancement of soil structure and water stability [5]. Scholars from foreign developed countries have also done various researches. For instance, Terry [6-8] has found that polyacrylamide can increase the stability of agglomerated particles and permeability of clay soil by 
$18 \%$ and 2 times respectively. Kukal's research has showed that the polyvinyl acetate can well improve the water stability of soil particles [9]. Meanwhile, both scholars at home and abroad have made numerous researches on the process of slope erosion and factors that affect the slope's resistance to erosion caused by rainfall. The process of slope erosion by indoor and field simulation test has reproduced that the main control factors that affect the slope's stability is rainfall intensity, duration and slope ratio [10-12].

This paper adopts the comprehensive prevention and protection method by ecological slope fixation. The method includes an interception and drainage system, a bamboo nail anchoring system, a lattice system for solidifying soil and a root reinforcement system. Based on the model test method, the effects of factors such as mixing amount of different modifying agent, lattice forms, rainfall intensity, duration and slope ratio on the slope's resistance to rainfall erosion are studied as well as the rules and evolutionary mechanism of soil mass erosion under these factors and their different combinations.

\section{Rainfall Erosion Stimulation Test}

The effects of factors, such as modified soil, lattice forms, rainfall intensity, duration and slope ratio, on the anti-erosion capacity of slope have been considered in the test. Modified soil has two types: plain soil and modifying agent-modified soil. Lattice forms also have two types as lattice with beams and without beams. There are three types of rainfall intensity, including $70 \mathrm{~mm} / \mathrm{h}, 100 \mathrm{~mm} / \mathrm{h}$ and $140 \mathrm{~mm} / \mathrm{h}$. For durations, there are six different lengths as $5 \mathrm{~min}, 10 \mathrm{~min}$, 15min, 25min, 35min and 45min. Three types of slope ratio are designed: 1:0.75, 1:1 and 1:1.25. Cross-over trial is applied, the results are shown in Table 1 . The model slot dimension is $30 \mathrm{~cm} \times$ $30 \mathrm{~cm}$. The actual slope created is $30 \mathrm{~cm} \times 20 \mathrm{~cm}$ with a depth of $6 \mathrm{~cm}$. The density of its surface protection soil is $1.1 \mathrm{~g} / \mathrm{cm}^{3}$, and moisture content is about $10 \%-15 \%$. The soil used in all stimulation tests is actually used in engineering. Then, reduce the dimensions of slopes and lattice forms based on a certain ratio, and compare the anti-erosion capacity of shallow slope under different working conditions qualitatively and semi-quantitatively. 
Table 1 Table of different working conditions

\begin{tabular}{|c|c|c|c|c|c|}
\hline No. & Plain Soil & Modified Soil & Lattice Beam & Slope Ratio & Rainfall $[\mathrm{mm} / \mathrm{h}]$ \\
\hline 1 & $\sqrt{ }$ & - & - & $1: 1$ & 70 \\
\hline 2 & - & $1 \%$ & - & $1: 1$ & 70 \\
\hline 3 & - & $2 \%$ & - & $1: 1$ & 70 \\
\hline 4 & - & $3 \%$ & - & $1: 1$ & 70 \\
\hline 5 & - & $4 \%$ & - & $1: 1$ & 70 \\
\hline 6 & - & $6 \%$ & - & $1: 1$ & 70 \\
\hline 7 & $\sqrt{ }$ & - & $\sqrt{ }$ & $1: 1$ & 70 \\
\hline 8 & - & $1 \%$ & $\sqrt{ }$ & $1: 1$ & 70 \\
\hline 9 & - & $3 \%$ & $\sqrt{ }$ & $1: 1$ & 70 \\
\hline 10 & $\sqrt{ }$ & - & - & $1: 1$ & 100 \\
\hline 11 & - & $3 \%$ & - & $1: 1$ & 100 \\
\hline 12 & $\sqrt{ }$ & - & - & $1: 1$ & 140 \\
\hline 13 & - & $3 \%$ & - & $1: 1$ & 140 \\
\hline 14 & $\sqrt{ }$ & - & - & $1: 0.75$ & 70 \\
\hline 15 & - & $3 \%$ & - & $1: 0.75$ & 70 \\
\hline 16 & $\sqrt{ }$ & - & - & $1: 1.25$ & 70 \\
\hline 17 & - & $3 \%$ & - & $1: 1.25$ & 70 \\
\hline
\end{tabular}

\section{Results and Discussion}

Modified Results and Optimal Mixing Amount. Scour velocity refers to the quantity of soil eroded of per unit area of slope in unit time, which reflects the scouring and eroding ability of rainfall runoff to slopes. Based on the data collected from working condition 1 to 6 , a change curve of scour velocity with rainfall duration is concluded as shown in Fig. 1. 


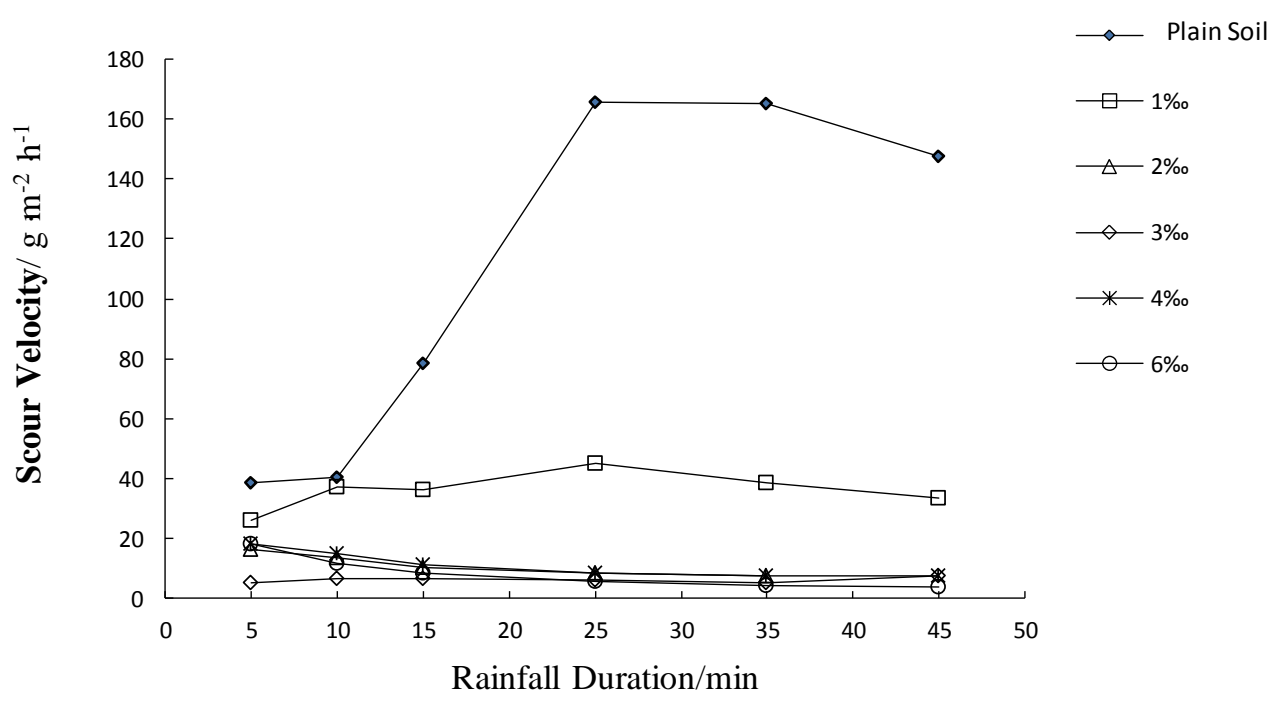

Figure 1. Finite Change curve of scour velocity with rainfall duration

It is obvious that the scour velocity of plain soil slope increases slowly at the beginning, but surges after 10 minutes and reaches its peak after 25 minutes, then slows down gradually. Combined with the test phenomenon, the slope is under splash erosion and sheet erosion, then moves into rill erosion. As these narrow rills converge, finally several paralleled trenches are formed down along with the slope, as shown in Fig. 2(a), when the scour velocity reaches its peak. During the period that stable trenches are forming on the surface of the slope, runoff mainly flows the tracks of trenches to the root of the slope, which results in decrease of scour velocity, as shown in the Fig. 2(b). Based on analysis, modifying agent can postpone the time when peak velocity occurs, or even eliminate that velocity. The scour velocity of modified soil with mixing amount of modifying agent over $2 \%$ decreases as the rainfall duration extends. It is considered that at the beginning of rainfall, the loose particles on the slope's surface are easily eroded by runoff. When loose particles reduce, the anti-erosion ability of modifying agent works, and the surface crusts, rainwater is prevented from soaking into the soil, as shown in Fig. 2(c). In a word, the scour velocity of modifying agent-modified soil slope is much slower than that of plain soil slope. Modifying agent-modified soil slope has a strong resistance to erosion, and such ability improved as the mixing amount of modifying agent increases.

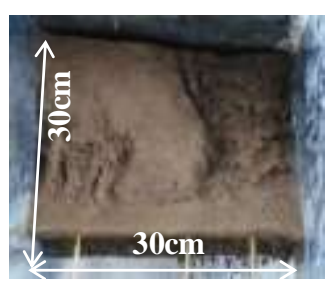

(a)

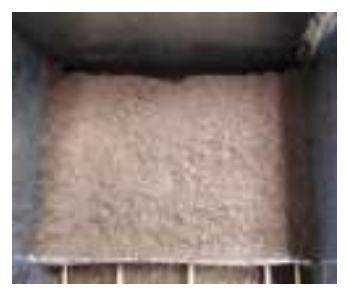

(d)

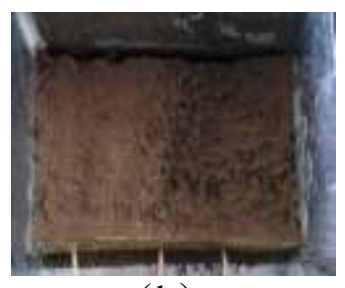

(b)

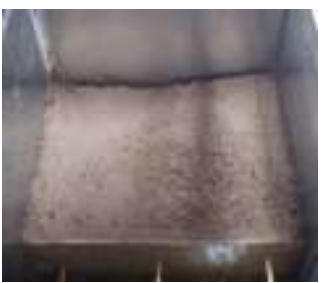

(e)

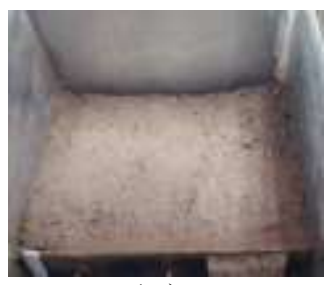

(c)

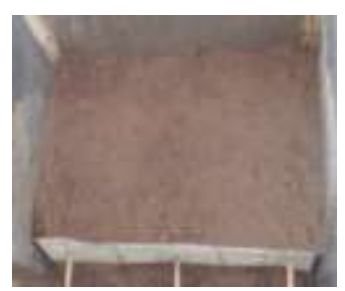

(f)

Figure 2. Finite Slope Erosion Situation after 45 Minutes 

( (a) Plain Soil
(b) $1 \% \mathrm{PMP}$
(c) $2 \% \mathrm{PMP}$
(d) $3 \%$ PMP
(e) $4 \%$ PMP
(f) $6 \%$ PMP )
$\alpha=\frac{w_{1}-w_{2}}{w_{1}} \times 100 \%$

In the above formula, $\alpha$ means the reduction rate of scour velocity, w1 refers to the scour velocity of plain soil slope, and w2 refers to the scour velocity of modified soil slope.

The reduction rates of scour velocity of slopes with different amount of modifying agent under different rainfall duration are shown in the Table 2. The data in Table 3 suggests that the reduction rate of scour velocity increases as the rainfall duration extends. The reduction rate of slope with $2 \%$ o of modifying agent reaches $90 \%$ after 25 minutes of rainfall, even slopes with $1 \%$ of modifying agent the reduction rates are over $70 \%$, which qualitatively suggests that the modifying agent-modified soil slope has a strong resistance to erosion, the performance grows significant especially under long-period scouring.

Table 2 Reduction rate of scour velocity

\begin{tabular}{ccccccc}
\hline \multirow{2}{*}{$\begin{array}{c}\text { Mixing Amount of } \\
\text { Modifying agent }\end{array}$} & \multicolumn{6}{c}{ Reduction Rate of Scour Velocity $\alpha(\%)$} \\
\cline { 2 - 7 } & $5 \mathrm{~min}$ & $10 \mathrm{~min}$ & $15 \mathrm{~min}$ & $25 \mathrm{~min}$ & $35 \mathrm{~min}$ & $45 \mathrm{~min}$ \\
\hline 1 & 32.4 & 7.1 & 53.7 & 72.9 & 76.6 & 77.1 \\
2 & 45.4 & 59.2 & 82.9 & 93.8 & 94.9 & 94.7 \\
3 & 83.4 & 83.2 & 91.3 & 96.2 & 96.7 & 95 \\
4 & 52.1 & 62.9 & 85.6 & 95 & 95.4 & 94.8 \\
6 & 52.5 & 70.6 & 89.4 & 96.5 & 97.2 & 97.4 \\
\hline
\end{tabular}

It also can be learned that in the earlier stage of rainfall, when the duration is the same, the reduction rate of scour velocity increases first, then decreases as the mixing amount of modifying agent increases, and the reduction rate meets its maximum with 3\%o of modifying agent. Under the same rainfall duration, after 25 minutes, the reduction rate generally increases first, then tends to be stable with the increase in mixing amount of modifying agent. The analysis shows that in the earlier stage of rainfall, the crust is gradually formed under the loose particles of modified slope's surface, and the crusting velocity increases with the mixing amount of the modifying agent. When a certain amout is reached, the surface of slope may crust too early to affect the infiltration rate of rainwater to some extent, and a relatively large runoff on the surface means enhanced ability of carrying the loose particles on the surface. Therefore, in the earlier stage, 3\%o of modifying agent will have the best results. As rainfall duration extands, when loose particles are all washed away and the surface gradually crusts, then the modified results of mixing amounts over $2 \%$ are basically the same. Therefore, based on the modified results of modifying agent in the tests, and the economoic factor, the recommended mixing amount of modifying agent shall be $3 \%$.

Modified Results of Lattice Beams to Slopes. Cumulate of loess erosion means the total weight of dried loess eroded by rainfall, which reflects the amount of soil erosion as the rainfall duration increases. According to working condition 1 (plain soil), working condition 2 (soil with 1\%o modifying agent), working condition 4 (soil with 3\%o modifying agent), working condition 7 (soil with lattice beams), working condition 8 (soil with $1 \%$ modifying agent and lattice beams), working condition 9 (soil with 3\%o modifying agent and lattice beams), Fig. 3 and Fig. 4 are made. 


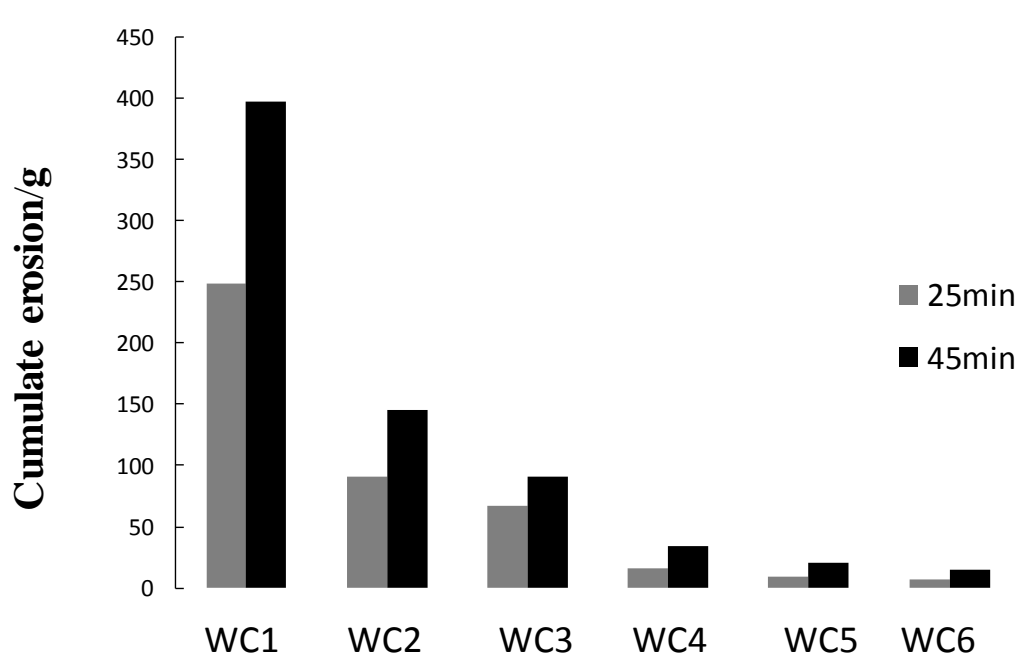

Figure 3. Finite Cumulant of loess erosion under different working conditions

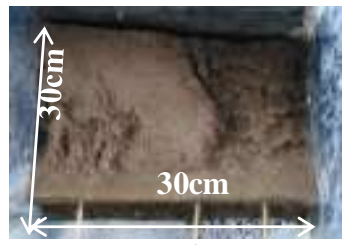

(a)

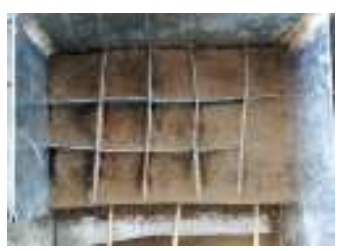

(d)

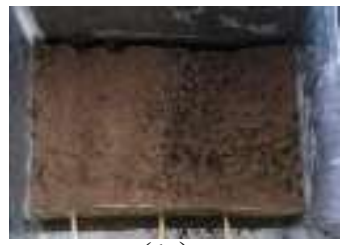

(b)

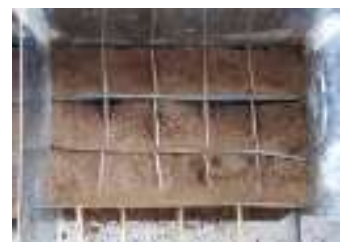

(e)

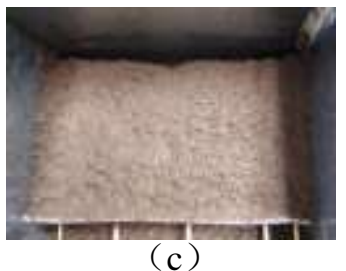

(c)

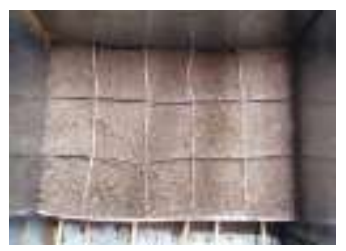

(f)

Figure 4. Finite Slope erosion condition after 45 minutes under different working conditions
( (a)working condition 1
(b) working condition 2
(c) working condition 4
(d) working condition 7
(e) working condition 8
(f) working condition 9 )

Comparing working condition 1 with 7 , working condition 2 with 8 , and working condition 4 with 9 , the results show that lattice beams can effectively diminish the erosion of slopes, reducing the amount of soil loss. After 45 minutes of rainfall, the cumulate erosion of plain soil slope, modified slope with $1 \%$ modifying agent and modified slope with $3 \%$ modifying agent that all use lattice beams reduces by $63 \%, 63 \%$ and $25 \%$ respectively than these without lattice beams, which qualitatively suggests that the adoption of lattice beam can greatly reduce the soil loss. It is analyzed that lattice beam deadens the erosion by slowing down the runoff velocity on the slope's surface.

To compare the improvement results of modifying agent (working condition 2 and 4), lattice beam (working condition 7) and the use of both (working condition 8 and 9), the reduction rate of cumulate erosion $\beta$ is introduced as the reducing amount of each working condition's cumulate erosion (represented by $\mathrm{m}$ ) compared to that of working condition 1 (plain soil slope, represented by $\mathrm{m} 0)$. Which is:

$$
\beta=\frac{m_{0}-m}{m_{0}} \times 100 \%
$$

Based on the above formula, the value of $\beta$ is calculated as shown in Table 3 . The data in Table 3 shows that modifying agent, lattice beam and the comprehensive measure by using both method can 
all effectively improve the water and soil loss of side slope, with the performance of comprehensive measure better than that of modifying agent and lattice beam separately. When mixing amount of modifying agent is increased to $3 \%$, the results of modifying agent are almost equal to that of comprehensive measure. In conclusion, based on the test results, a proper improvement measure can be adopted according to different working conditions, so as to ensure that the measure is economical and reliable.

Table 3 Reduction rate of cumulate erosion under various operating conditions

\begin{tabular}{lccccccc}
\hline $\begin{array}{l}\text { Working } \\
\text { Condition }\end{array}$ & $\begin{array}{l}\text { Plain Soil with } \\
\text { Lattice Beams }\end{array}$ & $\begin{array}{l}\text { Modified Soil with } \\
1 \% \text { Modifying } \\
\text { agent }\end{array}$ & $\begin{array}{l}\text { Modified Soil with } \\
\text { agent }\end{array}$ & $\begin{array}{l}\text { Modified Soil with } \\
\text { Modifying agent and Lattice } \\
\text { Beams }\end{array}$ & $\begin{array}{l}\text { Modified Soil with } \begin{array}{l}\text { Modifying agent } \\
\text { Lattice Beams }\end{array} \\
\text { and }\end{array}$ \\
\hline $25 \min \beta(\%)$ & 60 & 77 & 96 & 92 & 97 \\
\hline $\min \beta(\%)$ & 63 & & 77 & 94 & 91 & 96 \\
\hline
\end{tabular}

\section{Conclusions}

(1) Plain soil slopes are heavily eroded by rainfall. There is even collapse or landslide. The stability of the slope mass is poor. The simulation test shows that the separate and combined application of modifying agents and lattice beam can effectively enhance the anti-erosion capacity of the slope. The anti-erosion capacity increases as the amount of modifying agent increases. The longer the rainfall duration is, the better the effect of modifying agent is. Based on the decrease rate of erosion rate, it is put forward that the optimal mixing amount of modifying agent is $3 \%$.

(2) Slope with 3\% modifying agent is less affected by rainfall intensity and enjoys relatively strong anti-erosion ability. As the slope ratio increases, the slope mass tends to collapse suddenly. Therefore, the reasonable design of slope grade is a key factor influencing the deep layer stability and superficial layer slippery.

\section{References}

[1] H. M. Zhao, D. S. Jiang, et al: Journal of Hunan University (natural science edition), Vol. 31(5) (2004.) p.77-81. (In Chinese)

[2] X. M. Zhao, J. J. Shi, W. Wang, et al: Journal of Xian University of Engineering Science \& Technology. Vol.21 (2007) No.3 p.311-315 (In Chinese)

[3] Y. M. Liu. Effects of EN - 1 Ionic Stabilizer on Loess Soil and Ryegrass (Ph.D., Shanxi North West Agriculture and Forestry University, China 2013).

[4] J. Liu: Experimental Study on Development and Application of Polymer Soil Stabilizers. (Ph.D., Nanjing: Nanjing University, China 2011).

[5] H. J. Xia: Journal of soil and water conservation, Vol.14 (2000) No.3, p.14-17. (In Chinese).

[6] Z. Q. Li , C. Tang, R.L. Hu, et al: Journal of Civil Engineering and Management, Vol. 19(1) (2013) p. 9-15.

[7] Z. Q. Li , I. A. Oyediran, C. Tang, et al: Bulletin of Engineering Geology and the Environment, Vol. 73(4) (2014),p1013-1023.

[8] Z. Q. Li , C. Tang, R.L. Hu, et al: Environmental Earth Sciences, Vol.72(2)(2014),p363-371.

[9] Kukal S S, Kaur M, Bawa S S, et al: Catena, Vol. 70 (2007) No.3 p.475-479.

[10] G. W. Jia, L. T. Zhan and Y. M. Chen: Chinese Journal of Rock Mechanics and Engineering, Vol.28 (2009), No.9, p.1798-1803. (In Chinese)

[11] Y. Ding: Study on Stability of High Loess Slope under Artificial Rainfall Simulation. (MS., Northwestern University, China 2011).

[12] X. H. Huang, C. M. Wang, et al: Journal of Engineering Geology, Vol.23 (2015) No.4, p.725-730. (In Chinese) 